\title{
BNL -65184 \\ CoNf-980135. \\ TRANSIENT FREQUENCY-MODULATED SPECTROSCOPY: \\ APPLICATION TO THE MEASUREMENT OF VECTOR AND SCALAR CORRELATIONS IN MOLECULAR PHOTODISSOCIATION
}

\author{
Simon W. North \\ Department of Chemistry, Texas A\&M University, College Station, TX 77843-3255 \\ Gregory E. Hall \\ Chemistry Department, Brookhaven National Laboratory, Upton, NY 11973-5000
}

\begin{abstract}
An intrinsically time-resolved version of frequency-modulation (FM) spectroscopy has been recently developed and applied to the study of gas-phase photodissociation dynamics. Transient FM spectroscopy allows low background detection of radical species with shot-noise limited sensitivities, time resolution sufficient for detection of collisionless photoproducts, and frequency resolution characteristic of single-mode cw lasers. Methods for the quantitative analysis of Doppler-broadened FM line shapes to give velocity and rotational polarization information have been established permitting the measurement of scalar and vector properties of molecular fragmentation in exquisite detail. Several recent examples of the application of transient FM spectroscopy will be presented and discussed, including correlated scalar distributions in the dissociation of ketene from $\mathrm{CH}_{2}\left(\tilde{a}^{1} \mathrm{~A}_{1}\right)$ Doppler profiles, and the full vector correlation analysis of $\mathrm{CN}\left(X^{2} \Sigma^{+}\right)$fragments arising from ICN dissociation.
\end{abstract}

keywords: frequency-modulation (FM), Doppler spectroscopy, vector correlations, photodissociation dynamics

\section{Introduction}

The determination of the asymptotic scalar properties in photoinitiated chemical reactions has provided significant insight into many physical phenomena in photodissociation including non-adiabatic dynamics and intramolecular energy redistribution. Measurements of vector properties have revealed valuable information on the excited state symmetry and angular momentum polarization in molecular fragmentation. In the past decade there has been an emphasis on trying to measure correlated properties of dissociation which include correlations between various vector and scalar properties. Such detailed measurements represent an extremely precise description of the forces and torques responsible for the observed energy and angular momentum distributions. The global state distribution of one fragment is a sum over the states of the coincident fragment. Measurements of the full coincident state distribution may frequently be more informative than the individual uncorrelated state distributions when attempting to choose between approximate theoretical models of dissociation. There are presently many experimental techniques that permit the measurement of scalar and vector correlations in molecular fragmentation. These include state-selected ion imaging ${ }^{1}$, Doppler spectroscopy ${ }^{2}$, and neutral TOF tagging methods. ${ }^{3}$ In many of the early investigations at least one of the fragments was an atom. There have been relatively few examples of correlations where both fragments are at least diatomics.

Traditionally, Doppler spectroscopy has been performed using laser induced fluorescence (LIF) with subDoppler resolution pulsed lasers. Recently, we have demonstrated the advantages of measuring Doppler profiles using transient freqeuncy-modulation absorption spectroscopy. The technique employs single-mode cw probe lasers, avoiding all the uncertainties in accounting for imprecisely known laser linewidth effects. The analysis of Doppler lineshapes obtained by single-photon absorption is simple and can be accomplished with fewer excitation/detection geometries than for LIF. There have been two obstacles to implementation of frequency-modulation to nascent Doppler measurements. The first is the accurate determination of the absolute phase of the FM lineshapes, which contain absorption and dispersion components in quadrature. A precise knowledge of the phase angle permits the extraction of only the absorption component with negligible contamination from the dispersion, which dominates the far-off resonant signal. Even a modest contribution

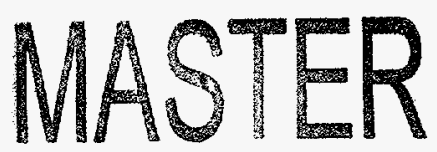


from the dispersion component, if unaccounted for, can result in large errors in the derived velocity distribution and vector correlations. We have developed a method which uses simultancous least-squares fitting of in-phase and quadrature FM signals from thermalized signals for a phase calibration. A linear combination of measurcd in-phase and quadrature spectra can then be constructed to eliminate the component of the line shape due to dispersion. The second obstacle has been the inversion of the finite difference FM signal to reconstruct the Doppler-broadened absorption profile in an intermediate regime where the laser linewidth is much less than the modulation frequency, yet the modulation frequency is comparable to the Doppler-broadened absorption linewidth. Although we initially relied on a recursion relation to recover the underlying Doppler-broadened absorption lineshape we have found an approximate method which displays better stability to noise. Based on these new developments it is now to possible generate high fidelity Doppler profiles from two-channel FM data.

\section{Experimental}

The details of the transient absorption FM spectrometer have been presented elsewhere. ${ }^{4.5}$ Briefly, the cw output of a Ar+ pumped Ti:sapphire ring laser is passed through an electro-optic modulator driven at $200 \mathrm{MHz}$. This results in three frequencies: the carrier, $\omega_{c}$, and two sidebands at $\omega_{\mathfrak{c}} \pm 200 \mathrm{MHz}$. The intensity of the sidebands is typically $<20 \%$ of the carrier. All three frequencies are attenuated with neutral density filters to avoid saturation of optical transitions and steered through a glass reaction cell containing a slow flow (4 SCCM) of precursor. The probe beam is overlapped with the output of a pulsed ultraviolet photolysis laser operated at $10 \mathrm{~Hz}$. There are three independent excitation/detection geometries for single photon detection. Geometries (1) and (2) refer to the cases where the probe and photolysis beams are collinear and the polarization are parallel and perpendicular, respectively. Geometry (3) involves orthogonal propagation directions with the photolysis polarization parallel to the probe beam propagation direction. Geometries (1) and (2) are implemented in a 1.5 $\mathrm{m}$ long cell; geometry (3) is implemented in a small multipass absorption cell with an active probed sample length about 10 times shorter. Differential absorption or dispersion of the sidebands is detected as a $200 \mathrm{MHz}$ beat note on a Si PIN photodiode. The rf is separated from the DC signal using a fast Bias $T$, filtered, and amplified. The instantaneous power in-phase and in-quadrature with local oscillator are measured with an I\&Q demodulator. The resulting two channels are $\mathrm{AC}$ coupled into a digital oscilloscope, averaged, and stored in a computer. The oscilloscope is triggered by a photodiode viewing the photolysis pulse, and 50-100 timedependent traces are averaged at each step of the probe laser frequency. The complete data set is a twodimensional array of FM signal vs. time and wavelength from which FM Doppler profiles at an arbitrary time delay after the photolysis laser pulse can be extracted and analyzed. The recovery of pure absorption Doppler lineshapes from two channel FM lineshapes has been the subject of a previous publication. ${ }^{5}$

We provide two figures that illustrate the two-dimensional nature of the raw FM data. Figure 1 shows a FM Doppler lineshape of $\mathrm{CN}(N=30, \mathrm{v}=0)$ arising from ICN photodissociation at $248 \mathrm{~nm}$. This lineshape was obtained from the full $2-D$ data set by extracting the frequency dependence at times within a $100 \mathrm{~ns}$ wide gate, directly following the UV laser pulse. Figure 2 shows the timedependent waveforms corresponding to several specific probe laser frequencies, indicated by the arrows in Figure 1. The selection of the time gates enables one to generate Doppler profiles at an arbitrary timedelay from a single measurement. This multiplexing has a number of important advantages over other techniques. For gascell experiments, it is possible to assess the effect of collisional relaxation on the lineshapes by generating Doppler profiles at sucessively earlier time gates until there is no detectable change in the lineshapes. This allows verification that the rep 7 ed Doppler profiles are nascent, without the need of

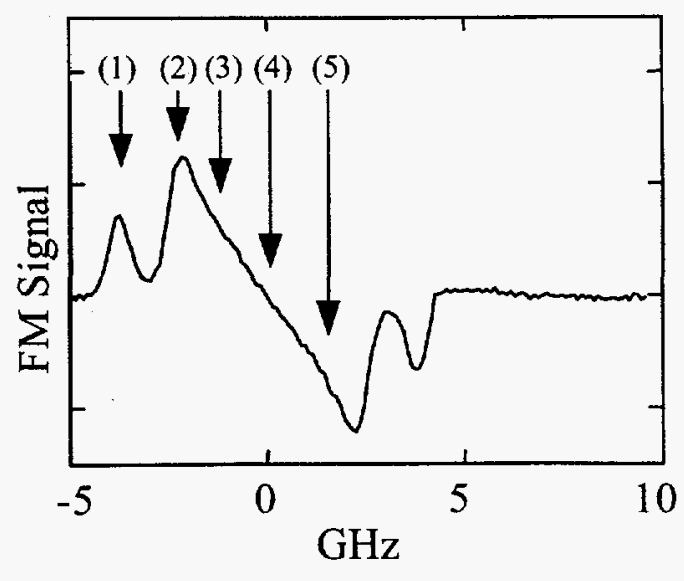

Figure 1. Nascent FM Doppler lineshape for $\mathrm{CN}(\mathrm{v}=0, N=30)$ from $\mathrm{ICN}$ photodissociation at $248 \mathrm{~nm}$. Arrows indicate the frequencies shown in Figure 2. 


\section{DISCLAIMER}

This report was prepared as an account of work sponsored by an agency of the United States Government. Neither the United States Government nor any agency thereof, nor any of their employees, makes any warranty, express or implied, or assumes any legal liability or responsibility for the accuracy, completeness, or usefulness of any information, apparatus, product, or process disclosed, or represents that its use would not infringe privately owned rights. Reference herein to any specific commercial product, process, or service by trade name, trademark, manufacturer, or otherwise does not necessarily constitute or imply its endorsement, recommendation, or favoring by the United States Government or any agency thereof. The views and opinions of authors expressed herein do not necessarily state or reflect those of the United States Government or any agency thereof. 


\section{DISCLAIMER}

Portions of this document may be illegible electronic image products. Images are produced from the best available original document. 
additional experiments. We have also found that global vibrational state distributions can be obtained easily by allowing the samples to undergo translational and rotational relaxation while remaining vibrationally nascent. It is also possible to check the experimental phase angle for each data array by analyzing a late-gate thermal Doppler lineshape. In addition, for spectroscopic investigations, recording the time-dependence of each spectral feature can aid in its assignment.

\section{Results}

We have recently applied transient frequency-modulation absorption spectroscopy (TFMAS) to a wide range of chemical systems. Initial implementation of the technique focused on the near-infrared spectroscopy of methylene $e^{6}$ and halogenated analoges ${ }^{7}$. The superior $\mathrm{S} / \mathrm{N}$ compared to conventional absorption spectroscopy allowed the detection of weak spectral features and facilitated spectral assignments. We have also shown the utility of TFMAS as an effective means for measuring the rates of gas-phase chemical reactions. The bimolecular rate constant for the reaction of $\mathrm{CN}$ with $\mathrm{C}_{2} \mathrm{H}_{4}$ at $298 \mathrm{~K}$ could be easily measured and agreed well with established literature values. ${ }^{8}$

Of more relevance to the present paper is the application of the technique to measuring nascent Doppler profiles of photofragments. To date, we have successfully demonstrated the potential of TFMAS for examining photofragment vector and scalar correlations. The first system studied was the unimolecular dissociation of cyanogen, NCCN (ref 9). Our reinvestigation of this prototype of statistical dissociation uncovered many important deviations from theoretical predictions including the existence of a moderate exit barrier and substantial v-J correlations suggestive of approximate $K$-conservation in the dissociation. We have also examined the photodissociation dynamics of $\mathrm{CH}_{3} \mathrm{COCN}$ (ref 10) and $\mathrm{C}_{2} \mathrm{H}_{3} \mathrm{CN}$ (ref 11 ). In this paper we choose to concentrate on more recent examples which exploit the advantages of the TFMAS technique. The ultraviolet photodissociation of ICN and $\mathrm{CH}_{2} \mathrm{CO}$ are respectively benchmarks for direct, multiple surface photodissociation, and barrierless statistical unimolecular dissociation. Our study of ICN is extensive and we present here only a representative portion of the data and analysis. A full report of the system is currently in preparation. Recent data on the $308 \mathrm{~nm}$ dissociation of ketene is preliminary and not completely analyzed, although we are encouraged by the quality of our results.

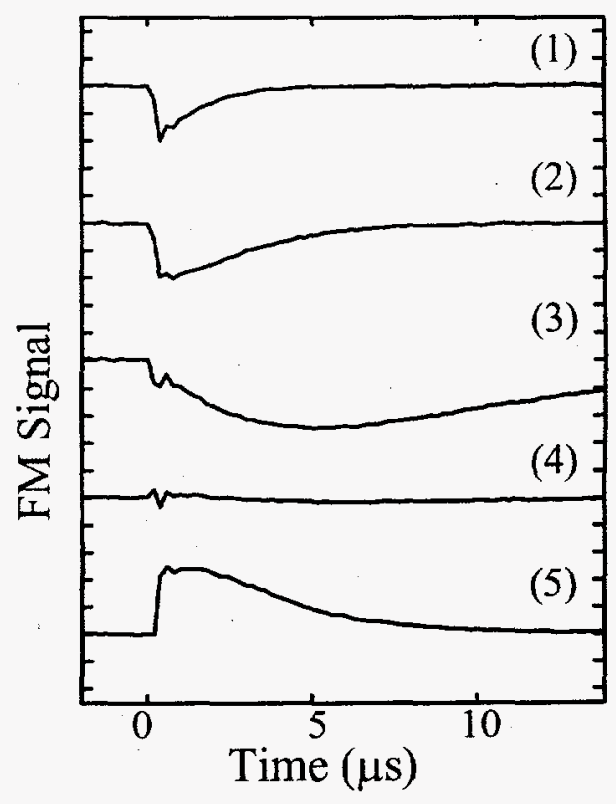

Figure 2. Single channel time-dependent traces of $\mathrm{CN}(v=0, N=30)$ corresponding to the probe frequencies in Figure 1.

\section{A. The Ultraviolet Photodissociation of ICN}

The ultraviolet photodissociation of ICN has become the prototype for multiple surface dynamics and has been extensively investigated by both experiment ${ }^{12}$ and theory. ${ }^{13}$ Optical absorption near $260 \mathrm{~nm}$ involves excitation to three optically bright states: ${ }^{3} \Pi_{0+},{ }^{1} \Pi_{1},{ }^{3} \Pi_{1}$. The ${ }^{3} \Pi_{0+}$ state is reached by a parallel transition and the ${ }^{1} \Pi_{1}$ and ${ }^{3} \Pi_{1}$ states are reached by perpendicular transitions. All three states are strongly bent in the Franck-Condon region and the latter two states split into $\mathrm{A}^{\prime}$ and $\mathrm{A}^{\prime \prime}$ components in non-linear geometries. It is an avoided crossing between the ${ }^{3} \Pi_{0^{+}}\left(4 A^{\prime}\right)$ and ${ }^{\prime} \Pi_{1}\left(5 A^{\prime}\right)$ surfaces which is responsible for the interesting non-adiabatic dynamics observed in this system. 
We have used the TFMAS technique to measure state selected Doppler profiles for nascent $\mathrm{CN}$ fragments arising from ICN photodissociation at $308 \mathrm{~nm}, 266 \mathrm{~nm}$, and $248 \mathrm{~nm}$. Our results at $308 \mathrm{~nm}$ have been recently published. ${ }^{14}$ ICN dissociation at wavelengths $>300 \mathrm{~nm}$ has been considered much simpler because only $\mathrm{I}^{2}\left(\mathrm{P}_{3 / 2}\right)$ is energetically accessible. Excitation to the lowest ${ }^{3} \Pi_{1}$ state which asymptotically correlates to ground state products was thought to dominate the transition. Trajectories originating on the ${ }^{3} \Pi_{1}\left(E^{\prime}\right)$ state must follow an adiabatic path to products. The complete set of vector correlations observable with linearly polarized single photon detection have been determined for several rotational states in $\mathrm{v}=0$. All detected states exhibited strong positive velocity anisotropy indicating that the initial optical excitation is predominantly a parallel transition. The result challenged

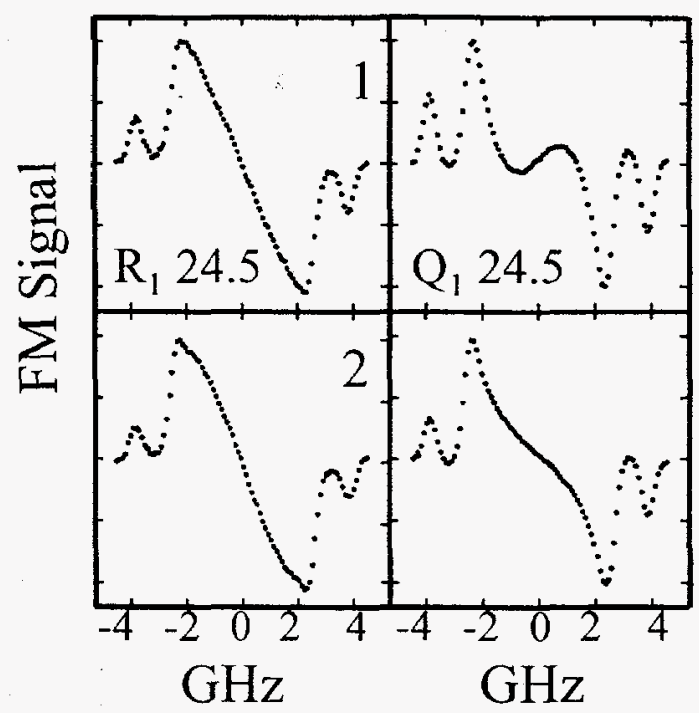

Figure 3. Raw FM Doppler lineshapes for $\mathrm{CN}(\mathrm{v}=0, N=24)$ from ICN photodissociation at $248 \mathrm{~nm}$. recent theoretical work which predicted that at wavelengths $>300 \mathrm{~nm}$ the perpendicular transition to the ${ }^{3} \Pi_{1}$ state should dominate the dissociation. From the fragment velocities and the bond dissociation energy, we can determine that hot bands dominate the absorption at $308 \mathrm{~nm}$, providing further clues as to the nature of the red wing absorption in a thermal sample.

We have since extended our study of ICN photodissociation to $266 \mathrm{~nm}$ and $248 \mathrm{~nm}$. Although extensive $\mathrm{CN}$ Doppler spectra have been recorded at these wavelengths previously, recent theoretical calculations have highlighted several discrepancies with experimental results and have made some interesting predictions. A combination of the advantages from the TFMAS technique and probing the $\mathrm{CN}$ fragments via the $A-X$ transition has produced a highly detailed set of experimental data. There are several factors make the $A-X$ transition more favorable than the traditional $B-X$ transition used in LIF experiments. The presence of Q-branch transitions to compare with $\mathrm{P}$ - or R-branch lineshapes adds greatly to the stability of the vector correlation analysis, particularly for the determination of the $\mathbf{v} \cdot \mathbf{j}$ correlation. In addition, the $F_{l}$ and $F_{2}$ spin components, resolvable only at high $N$ for the $B-X$ transition, are well separated in the $A-X$ spectrum. The simplifications afforded by the $A-X$ transition, the higher spectral resolution of the $\mathrm{cw}$ probe laser, and the exquisite signal-to-noise characteristic of the TFMAS technique permit a full polarization analysis of the nascent Doppler lineshapes, even for those lines which have contributions from both $I$ and $I^{*}$ coincident channels.

Figure 3 shows nascent single channel FM lineshapes for $\mathrm{CN}(N=24, v=0)$ arising from the ICN photodissociation at $248 \mathrm{~nm}$. The data were collected in the collinear photolysis/probe geometries and (1) and (2). The single scans were acquired with 50 shot averaging at each $100 \mathrm{MHz}$ step of the probe laser. Contributions from both the I and I* channels are clearly resolved. The $F_{1}$ and $F_{2}$ spin rotation components, separated by the spin-orbit splitting in the $A-X$ transition, are completely isolated. The overlap of the $F_{1}$ and $F_{2}$ components in the B-X transition precluded complete polarization analysis of $\mathrm{CN}$ states $N<50$. Analysis is conducted on phase corrected and reconstructed FM signals.

The Doppler profiles were analyzed using the bipolar moment formalism of Dixon for the case of linearly polarized one-photon absorption spectroscopy. ${ }^{15}$ The Doppler profile for an ensemble with a single speed, $v$, can be written as

$$
D(w)=\frac{1}{2 v}\left[g_{0}+g_{2} P_{2}\left(\frac{w}{v}\right)+g_{4} P_{4}\left(\frac{w}{v}\right)\right]
$$


where $w$ is the component of the laboratory velocity along the probe direction and $P_{2}$ and $P_{4}$ are Legendre polynomials. The coefficients $g_{i}$ depend on geometrical factors, the rotational branch, and five non-trivial renormalized bipolar moments of the correlated velocity and angular momentum distribution. The Doppler profile for each $I\left({ }^{2} \mathrm{P}_{\mathrm{J}}\right)$ component has this form with its own fixed value of $v$. Since we perform our experiments on a room temperature ensemble, we include a thermal average over parent internal states and a convolution over the parent thermal velocity distribution. We simultaneously fit a set of six Doppler profiles, consisting of R- and Q-branches measured in three independent geometries, to determine the full set of bipolar moments and an $I / I^{*}$ branching ratio. The values of the center-of-mass bipolar moments and branching ratio are iteratively adjusted to minimize the $\chi^{2}$ of the entire data set. Figure 4 shows a set of $\mathrm{Q}_{1} 50.5 \mathrm{CN}(\mathrm{v}=0)$ Doppler profiles in the three experimental geometries at $248 \mathrm{~nm}$. The solid line is the best global fit to the data, including R-branches not shown.

A careful analysis of the translational energy release as a function of $\mathrm{CN}$ state has re-established a $\mathrm{I}-\mathrm{CN}$ bond dissociation energy of $26,600 \pm 200 \mathrm{~cm}^{-1}$ in good agreement with the value of Wittig and co-workers ${ }^{16}$ but significantly different from the $28,700 \pm 100 \mathrm{~cm}^{-3}$ recently advocated. ${ }^{17}$ Table I lists the complete set of bipolar moments from the analysis of several high $N \mathrm{CN}(\mathrm{v}=0)$ states at $266 \mathrm{~nm}$ and 248 $\mathrm{nm}$. There are several important results that differ from previous measurements. The analysis is most sensitive to the values of $\beta_{0}^{2}(20)$, which is one half of the velocity anisotropy parameter, $\beta$, and $\beta_{0}^{\prime}(22)$, the $\mathbf{v}-\mathbf{j}$ correlation. In the limit of prompt recoil the anisotropy parameter ranges from 2.0 for a parallel transition to -1.0 for a perpendicular transition. Since the dissociation is rapid, the non-limiting anisotropy parameters primarily reflect the relative contributions of parallel and perpendicular transitions resulting in the detected final state. Our results at $266 \mathrm{~nm}$ show a clear trend for an increase in the anisotropy of the I channel with $N$. This dependence is a result of the high degree of rotational

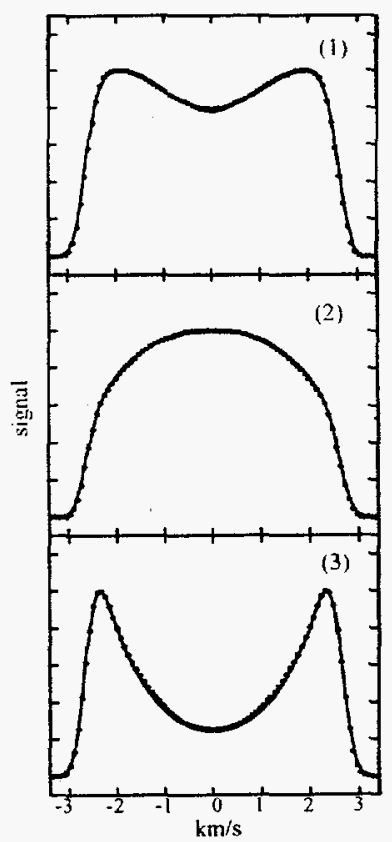

Figure 4. Q-branch Doppler profiles for $\mathrm{CN}(\mathrm{v}=0, N=50)$ from ICN photodissociation at $248 \mathrm{nun}$. The Solid line is the best global fit to the data including R-branch data.

Table 1 Bipolar Moments for selected $\mathrm{CN}$ states formed in coincidence with $I\left({ }^{2} \mathbf{P}_{3 / 2}\right)$

\begin{tabular}{l|lllll} 
& $\beta_{0}^{2}(02)$ & $\beta_{0}^{2}(20)$ & $\beta_{0}^{0}(22)$ & $\beta_{0}^{2}(22)$ & $\beta_{0}^{2}(42)$ \\
\hline $266 \mathrm{~nm}$ & & & & & \\
$N=40$ & $-0.14 \pm 0.08$ & $0.57 \pm 0.03$ & $-0.42 \pm 0.05$ & $0.32 \pm 0.06$ & $-0.23 \pm 0.08$ \\
$N=46$ & -0.09 & 0.62 & -0.41 & 0.32 & -0.26 \\
$N=50\left(F_{1}\right)$ & -0.32 & 0.68 & -0.39 & 0.38 & -0.24 \\
$N=50\left(F_{2}\right)$ & -0.29 & 0.67 & -0.38 & 0.35 & -0.26 \\
$N=55$ & -0.36 & 0.74 & -0.43 & 0.38 & -0.22 \\
$248 \mathrm{~nm}$ & & & & & \\
$N=40$ & -0.09 & 0.36 & -0.35 & 0.20 & -0.10 \\
$N=50\left(F_{l}\right)$ & -0.20 & 0.45 & -0.39 & 0.30 & -0.22 \\
$N=50\left(F_{2}\right)$ & -0.18 & 0.49 & -0.41 & 0.27 & -0.22 \\
\hline
\end{tabular}


excitation in those trajectories that originate on the ${ }^{3} \Pi_{0+}$ surface and evolve adiabatically to give $I$. This effect has been predicted by theory but not detected in a previous study by Wittig and co-workers. ${ }^{16}$ We observe the opposite effect for the I* channel, a trend that has also been predicted, although in less precise agreement with current measurements. At $248 \mathrm{~nm}$, qualitatively similar trends have been previously reported, ${ }^{12}$ although we still find quantitative discrepancies.

We have also determined the state-dependent $\mathrm{I} / \mathrm{I}^{*}$ branching ratios and found excellent agreement with earlier work for $266 \mathrm{~nm}$. At $248 \mathrm{~nm}$, however, we find significantly more $\mathrm{I}^{*}$ in coincidence with lower $\mathrm{CN}$ rotational states compared to previous reports. The differences arise from a more stable analysis of the vector properties of the I channel for those rotational states with significant contributions from both I an $I^{*}$ channels. Our results are closer to recent calculations by Morokuma and co-workers. ${ }^{18}$ Figure 5 shows the state-dependent branching ratios determined in the present work (solid circles), previous experimental work (open circles), and theoretical results at $248 \mathrm{~nm}$ (dashed line). The solid line is spline fit to our measurements. Using our results at $248 \mathrm{~nm}$ and the rotational state distribution ${ }^{12}$ we obtain a overall $\mathrm{l} / \mathrm{I}^{*}$ branching ratio of $0.51 \pm 0.02$, slightly outside the error limits of the previous experimental result of $0.43 \pm 0.04$.

Our measured Doppler profiles have sufficient signal-to-noise to detect small variations in the available energy with $N$. We find that assuming a thermal distribution of parent internal states provides excellent fits for most $N$-states of the I channel but only

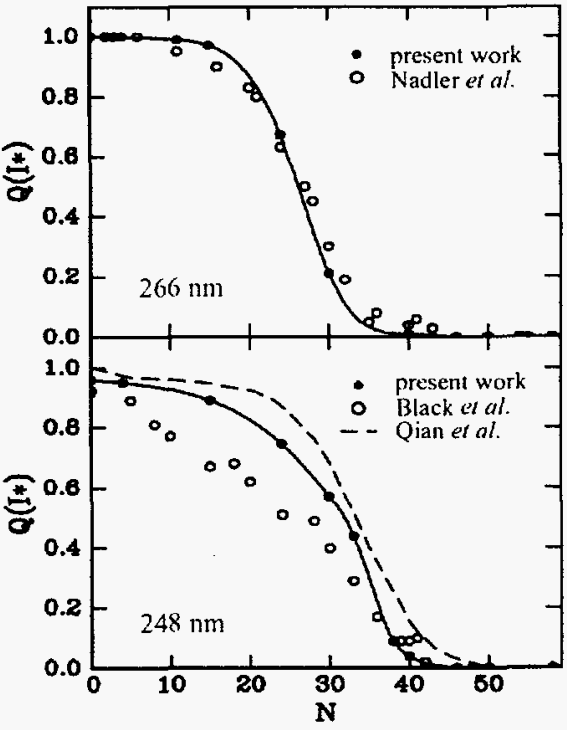

Figure 5. Plot of the $I^{*}$ fraction vs. $N$ for ICN photodissociation at 248 and $266 \mathrm{~nm}$. for low $N$-states $(<15)$ of the I* channel. Satisfactory fits for the high $N$ states of the $\mathrm{I}^{*}$ channel can be obtained by suppressing the contribution of the vibrationless parent molecules in the thermal averaging. The observation indicates the strong influence of initial parent bending on producing high $N \mathrm{CN}$ in coincidence with $\mathrm{I}^{*}$. Most of the $\mathrm{I}^{*}$ products arise from trajectories which start from the ${ }^{3} \Pi_{0+}$ state and remain diabatic. The low rotation for these products is a result of the bending force constant for the ${ }^{3} \Pi_{0+}$ state which becomes positive at moderate $R_{I-C N}$. Calculations have shown that for these trajectories parent bending motion results in larger product rotation.

There have been numerous previous discussions of the possibility and implications of photofragment $\mathbf{v} \cdot \mathbf{j}$ correlations, $\beta_{0}^{\prime \prime}(22)$, deviating significantly from -0.5 in the case of linear triatomic dissociation. We typically find values of $\beta_{0}^{\circ}(22)=-0.3$ following $308 \mathrm{~nm}$ photodissociation, and -0.4 at $266 \mathrm{~nm}$ and $248 \mathrm{~nm}$. In the limit of zero total angular momentum, the problem is nearly trivial. Neglecting the angular momentum of the dissociation photon and the iodine atom compared to high angular momentum of a diatomic fragment, angular momentum conservation requires that the orbital angular momentum of the fragment pair, $l$, and the rotational angular momentum of the diatom, $j$, be antiparallel and equal in magnitude. Since $\mathbf{I}$ is perpendicular to the center-of-mass relative velocity, $\mathbf{v}$, a sharply perpendicular relation between $\mathbf{v}$ and $\mathbf{j}$ is required. More generally, angular momentum conservation strictly forbids $\mathbf{j}$ to have any component along $\mathbf{v}$ in excess of the algebraic sum $\mathbf{j}_{\text {atom }}+\mathbf{J}_{\mathrm{parent}}+1$. This restriction on the molecular helicity can still allow for significant depolarization without violating angular momentum conservation. A phase space theory prediction for the $\mathbf{v} \cdot \mathbf{j}$ correlation of $\mathrm{CN} \mathrm{v}=0, N=30$, for example, is -0.1 for a room temperature initial distribution of ICN rotational states. In order for this statistical degree of depolarization to be achieved, however, all allowed helicity states of the recoiling pairs must be accessible, starting from bound states with body-fixed angular momentum projection numbers $\Omega=0$ or 1 only. The $\mathrm{CN} \mathbf{v} \cdot \mathbf{j}$ correlation thus gives a direct experimental measure of the number of asymptotic helicity states contributing to the photodissociation. An observed $\mathbf{v} \cdot \mathbf{j}$ correlation moment of -0.35 at $N=30$ could, for example, result from equal contributions from all total helicity states up to \pm 16 , or from a Gaussian distribution of helicity states characterized by a sigma of 10 . The reprojection of the initial body-fixed 
fragment angular momentum onto the final recoil velocity can be thought of as a Coriolis mixing of helicity states in the exit channel, which depends on the deviations from the axial recoil limit. These become more significant at high $\mathrm{J}$ and for lower translational energy channcls.

\section{B. The $308 \mathrm{~nm}$ Photodissociation of $\mathrm{CH}_{2} \mathrm{CO}$}

The unimolecular dissociation of ketene, $\mathrm{CH}_{2} \mathrm{CO}$, has been extensively studied and reviewed. ${ }^{19}$ It is considered one of the most important benchmark systems for understanding statistical models of dissociation. Significant understanding has been largely due to the early and ongoing efforts by Moore and co-workers who determined product state distributions and formation rates for individual quantum states as a function of photolysis wavelength. Near the singlet dissociation threshold the product state distributions and reaction rates are well described by phase space theory (PST) which represents the 'loosest' of possible transition states.

$$
\mathrm{CH}_{2} \mathrm{CO} \rightarrow \mathrm{CH}_{2}\left(\mathrm{à}^{1} A_{1}\right)+\mathrm{CO}
$$

As the excitation energy is increased the transition state tightens and modeling the experimental data requires more sophisticated theoretical treatments. Several theoretical models, notably variational RRKM theory, have now reproduced the vast majority of experimental measurements.

At energies above the threshold for the formation of $\mathrm{CO}(\mathrm{v}=1)$ the $\mathrm{CO}$ vibrational distribution is not well fit by PST, but the separate statistical ensembles model (SSE) provides excellent agreement with experiment. Modification of the PST correlated product state distribution within the SSE model is straightforward, applying a single scaling factor to each correlated vibrational distribution while leaving each correlated rotational distribution unaffected. Recent reports at $308 \mathrm{~nm}$ have suggested that the situation may be more complicated. ${ }^{6}$ For a low $\mathrm{CH}_{2}(0,0,0)$ rotational state, simulation of the Doppler profile required 2-3 times as much $C O(v=1)$ as the SSE prediction. Since the global population of $\mathrm{CO}$ in $\mathrm{v}=1$ agrees with SSE, the results would require a deficiency in coincident $\mathrm{CO}(v=1)$ for higher states of $\mathrm{CH}_{2}$. We have collected a superior set of Doppler spectra for a wide range of $\mathrm{CH}_{2}(0,0,0)$ rotational states both above and below the energetic threshold for $\mathrm{CO}(v=1)$. Figure 6 shows $\mathrm{FM}$ Doppler lineshapes for three $\mathrm{CH}_{2}(0,0,0)$ rotational states. The contribution from the slower $\mathrm{CO}(v=1)$ coincident channel is clearly observed for the $\left(1_{10}\right)$ state. This feature is absent in the $\left(6_{52}\right)$ state which is well above the $\mathrm{CO}(\mathrm{v}=1)$ threshold. We are optimistic about determining state-dependent vibrational branching ratios for this important reaction and assessing the ability of the SSE model to describe correlated vibrational distributions. Forward convolution simulations are currently in progress.

\section{Conclusions}

In summary, the application of TFMAS to the measurement scalar and vector correlations in molecular photodissociation is extremely promising. Despite the lower intrisic sensitivity compared to the competitive LIF technique we find that in practice superior Doppler profiles can often be obtained.

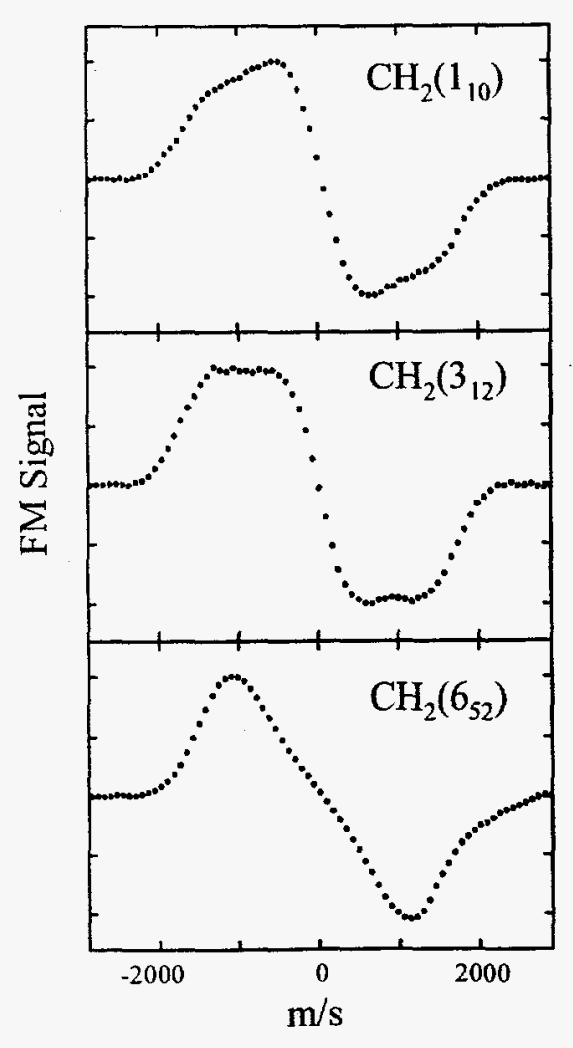

Figure 6. Nascent FM Doppler lineshapes of $\mathrm{CH}_{2}$ fragments from $\mathrm{CH}_{2} \mathrm{CO}$ photodissociation at $308 \mathrm{~nm}$ 
Furthermore, there are significant advantages in TFMAS including narrow probe laser linewidth, cw detection permitting time-wavelength multitasking, and ease of subsequent analysis. We have measured qualitative and quantitative differences with past experiments for the UV photodissociation of ICN. We have now measured detailed correlated information for a number of important chemical systems demonstrating the power of the method. Our work continues, specifically on the narrow band tunable photodissociation of NCCN.

The future looks bright. Experiments using low cost tunable diode lasers to replace the Ti:sapphire probe laser are in progress. This should permit a wide range of chemical species to be probed with greater simplicity and affordability. Combining the TFMAS technique with pulsed slit jets should provide key improvements to the experiment. The application of jet-cooled TFMAS to high-resolution spectroscopy

has already been demonstrated. ${ }^{20}$ Applied to the measurement of Doppler spectroscopy the technique should allow more careful control of the parent internal energy distribution for systems that lack wellresolved features in the absorption spectrum. The role of vibrational hot-bands in the photodissociation of ICN, for example, can be explored by comparing jet and room temperature Doppler profiles. The slit-jet also removes the initial parent velocity contribution to the Doppler profiles and eliminates the noisy mathematical deconvolution procedure from the analysis.

\section{Acknowledgments}

This work was performed at Brookhaven National Laboratory under Contract No. DE-AC0276CH00016 with the U.S. Department of Energy and supported by its Division of Chemical Sciences, Office of Basic Energy Sciences.

\section{References}

'M. Mons and I. Dimicoli, Chem. Phys. Lett. 131, 298 (1986).

${ }^{2}$ J. L. Kinsey, J. Chem. Phys. 66, 2560 (1977).

${ }^{3}$ C. G. Morgan, M. Drabbles, and A. M. Wodtke, J. Chem. Phys. 104, 7460 (1996).

${ }^{4}$ J. C. Bloch, R. W. Field, G. E. Hall, and T. J. Sears, J. Chem. Phys. 101, 1717 (1994).

${ }^{5}$ S. W. North, X. S. Zheng, R. Fei, and G. E. Hall, J. Chem. Phys. 104, 2129 (1996).

${ }^{6}$ B. - C. Chang, M. Wu, G. E. Hall, and T. J. Sears, J. Chem. Phys. 101, 9236 (1994).

${ }^{7}$ B. -C. Chang and T. J. Sears, J. Chem. Phys. 102, 6347 (1995); B. -C. Chang and T. J. Sears, J. Chem. Phys. 105, 2135 (1996); A. J. Marr, S. W. North, T. J. Sears, L. Ruslen, and R. W. Field, J. Mol. Spec. in press, (1998).

${ }^{8}$ S. W. North, R. Fei, T. J. Sears, and G. E. Hall, Int. J. Chem. Kinetics 29, 127 (1997).

${ }^{9}$ S. W. North and G. E. Hall, J. Chem. Phys. 106, 60 (1997); S. W. North and G. E. Hall, Ber. Bunsenges. Phys. Chem. 101, 496 (1997).

${ }^{10}$ S. W. North, A. J. Marr, A. Furlan, and G. E. Hall, J. Phys. Chem. A 101, 9224 (1998).

${ }^{11}$ S. W. North and G. E. Hall, Chem. Phys. Lett. 263, 148 (1996).

12 J. F. Black, J. R. Waldeck, and R. N. Zare, J. Chem. Phys. 92, 3519 (1990) and references therein.

${ }^{13}$ Y. Amatatsu, S. Yabushita, and K. Morokuma, J. Chem. Phys. 100, 4894 (1994) and references therein.

${ }^{14}$ S. W. North, J. A. Mueller, and G. E. Hall, Chem. Phys. Lett. 276, 103 (1997).

${ }^{15}$ R. N. Dixon, J. Chem. Phys. 85, 1866 (1986).

${ }^{16}$ I. Nadler, D. Mahgerefteh, H. Reisler, and C. Wittig, J. Chem. Phys. 82, 3885 (1985).

${ }^{17}$ K. Chen, C. Kuo, M. Tzeng, M. Shian, and S. Chung, Chem. Phys. Lett. 221, 341 (1994).

${ }^{18}$ J. Qian, D. J. Tannor, Y. Amatatsu, and K. Morokuma, J. Chem. Phys. 101, 9597 (1994).

${ }^{19}$ W. H. Green, C. B. Moore, and W. F. Polik, Ann. Rev. Phys. Chem. 43, 591 (1992).

${ }^{20}$ B. - C. Chang and T. J. Sears, Chem. Phys. Lett. 256, 288 (1996). 